Alcaraz Salarirche, N., Fernández Navas, M. y Pérez Granados, L. (2019) Principios de procedimiento y escenarios reales en la formación inicial de docentes. Aula de Encuentro, nº 21, volumen 1, pp. 123-142.

\title{
PRINCIPIOS DE PROCEDIMIENTO Y ESCENARIOS REALES EN LA FORMACIÓN INICIAL DE DOCENTES
}

\section{Principles of procedures and real situations in training teachers program}

\author{
Noelia Alcaraz Salarirche \\ Manuel Fernández Navas \\ Laura Pérez Granados
}

\begin{abstract}
Resumen
Los autores plantean que el eje principal del trabajo de los docentes en sus aulas es el diseño de actividades educativas. Sin embargo, reconocen que esto representa un problema ya que, en la formación inicial se abarca una gran cantidad de contenido teórico relevante para el ejercicio profesional, que pocas veces es trasladado a un contexto práctico donde los estudiantes tengan la posibilidad de poner en acción la teoría. La experiencia que se relata surge de esta preocupación y describe parte del proyecto realizado en una asignatura del Grado de Educación Infantil de la Universidad de Cádiz, donde el eje principal de la misma fue diseñar escenarios de aprendizajes reales que respetaran los principios teóricos trabajados durante el semestre. De este modo los futuros docentes debían planificar, a través de principios de procedimiento, una serie de
\end{abstract}


actividades para realizar con alumnado de Educación Infantil de dos colegios de la provincia.

\title{
Palabras Clave
}

Innovación, reflexión, formación inicial, principios de procedimiento, diseño didáctico.

\begin{abstract}
The authors state that the main focus of the work of teachers in their classrooms is the design of educational activities. However, they recognize that this represents a problem, since initial training covers a large amount of theoretical content relevant to professional practice, which is rarely translated into a practical context where students have the possibility to put into action the theory. The experience in this work arises from this concern and describes part of the project carried out in a subject of the kindergarten Training Teachers program of University of Cádiz, where the main axis of it was to design real learning scenarios that respect the theoretical principles worked during the semester. In this way, the future teachers had to plan, through the principles of procedure, activities to be carried out with children from kindergartens of two schools of the province.
\end{abstract}

\section{Key Words}

Innovation, reflection, training teachers, principles of procedures, didactic design.

\section{Introducción}

Este trabajo parte de dos cuestiones que preocupan a los autores: La primera es si el alumnado de los Grados de Educación, sabe tomar decisiones sobre el diseño y planificación de actividades alternativas al modelo que han vivido en su etapa escolar. Y la segunda: ¿qué hacemos para lograrlo? Para dar respuesta a ellas, partimos de una premisa: la tarea fundamental que debe realizar un docente es la de diseñar actividades educativas respaldadas por el cuerpo de conocimiento teórico que se debe empezar a construir en la formación inicial. Por tanto, debemos replantearnos, por una parte, qué modelos de programación empleamos y, a su vez, ofrecemos al futuro profesorado. $Y$ por otra, qué experiencias brindamos a nuestro alumnado para que pueda poner en juego los principios teóricos que se trabajan desde una determinada asignatura. 
A lo largo de nuestra trayectoria docente hemos detectado un problema que persiste en los Grados en Educación: la disonancia que hay entre las teorías que el alumnado maneja en sus discursos y el modo en que las formalizan y traducen al diseño de actividades reales. Realidad que responde en buena parte al modelo de programación por objetivos que persiste en las aulas y que poco ayuda a la toma de decisiones para planificar actividades con sentido educativo (Gertrudix, 1999), así como a la escasez de oportunidades prácticas que se ofrecen a los futuros docentes para diseñar actividades reales acordes a la teoría. De ahí que propongamos un análisis sobre los principales problemas que plantea el viejo modelo, y que planteemos la necesidad de ponerlo en entredicho, aportando como posible solución la inclusión de los principios de procedimiento como una alternativa a dicho modelo. Un modelo que, bajo el reto de planificar actividades reales con sentido educativo, sirva de guía a la hora de tomar decisiones didácticas; superando así esa desconexión que el alumnado tiene entre la teoría y la práctica.

\section{Del dicho al hecho... entre la teoría y la práctica}

Si pensamos en el trabajo que un docente realiza a lo largo de su vida profesional, veremos que dedica la mayor parte del tiempo a diseñar y poner en práctica actividades para su alumnado. De manera parecida lo expresa Salinas (2001) al decir que:

No olvidemos que la relación entre enseñanza y aprendizaje no es de causalidad, la enseñanza no siempre produce el aprendizaje, la enseñanza trata de crear las mejores condiciones para que el aprendizaje tenga lugar, por tanto se trataría de una especie de hipótesis que ponemos a prueba. Consecuentemente, podríamos pensar que es en las actividades en el aula donde debiera recaer el mayor peso de nuestra responsabilidad profesional a la hora de decidir aquello que "vale la pena» hacer y aquello que "vale la pena» evaluar (p. 49).

Por tanto, es de la calidad educativa de esas actividades, de la que depende, en gran medida, las posibilidades de aprendizaje del alumnado. Por lo que entendemos que, tanto en la formación inicial como permanente, cobra gran importancia el diseño didáctico. Así lo manifiestan Fernández Navas y Alcaraz Salarirche (2014), citado en Pérez Granados (2018), cuando afirman que la calidad de la labor profesional de un docente reside en la capacidad de poner en marcha las estrategias didácticas adecuadas para ofrecer a su alumnado actividades con sentido que les ayude en su desarrollo personal, social y profesional.

Dado el contexto en el trabajamos, creemos que es desde las universidades desde donde debemos formar al alumnado lo mejor posible para que, en un futuro, sepa cómo afrontar el diseño de actividades educativas y de calidad. Como afirman Sierra y Caparrós (2017) "la educación es un asunto complejo que no se resuelve con entrenamiento en competencias técnicas, por lo que desde la formación del magisterio debemos trabajar más allá" (p. 69). 
A este respecto, el cometido de los planes de estudio ha sido contemplar una gran cantidad de contenido teórico, en buena parte relevante, que el profesorado universitario debe manejar para decidir y programar qué hace con su futuro alumnado. Conceptos como aprendizaje, diversidad, evaluación, así como autores o autoras relevantes en estos campos, son objeto de estudio a diario en todas nuestras facultades de educación con la intención de que los maestros y las maestras que de allí salen, utilicen ese contenido teórico en el ejercicio de su profesión.

Sin embargo, a lo largo de nuestra experiencia venimos detectando que cuando le pedimos al alumnado que se está formando, que diseñe actividades, no recurre para ello a los conceptos estudiados; lo cual nos recuerda a lo que plantea Schön:

Los centros superiores de formación, inmersos en la angustia de los ataques externos y de las propias dudas internas, se hacen más y más conscientes de los problemas que tienen que ver con determinados supuestos fundacionales de los que han dependido siempre para su credibilidad y legitimidad. Han asumido que la investigación académica da como resultado un conocimiento profesional útil y que el conocimiento profesional que se enseña en los centros prepara a los estudiantes para las demandadas de la práctica en la vida real. Ambas creencias se cuestionan cada vez más (1987, pp. 22-23).

Existe una profunda disonancia entre lo que piensan sobre educación como fruto del trabajo con y sobre la teoría, y cómo formalizan el diseño de actividades, esto es, entre las "teorías proclamadas" y las "teorías en uso" (Argyris, 1993, citado en Pérez Gómez, Soto y Serván, 2015). De modo que, mientras el bagaje teórico suele ser bastante rico, elaborado y se nutre de gran cantidad de contenidos propios de las diferentes materias que han cursado en la universidad, en el segundo caso les cuesta, mucho, ir más allá de las actividades que han experimentado ellos mismos durante toda su escolaridad. Lo cual nos lleva a reflexionar sobre si desde los Grados en Educación estamos formando estudiantes que reproducen contenidos teóricos pero que encuentran enormes dificultades a la hora de trasladar dicha teoría a una situación práctica como, por ejemplo, el diseño de una actividad educativa.

Por lo tanto, lo que vamos a proponer en este artículo es trabajar con el alumnado en formación -a partir de una situación real- el modelo de principios de procedimientos como estrategia para planificar, con coherencia, actividades didácticas que han de llevar a cabo con estudiantes reales y sobre las que han de reflexionar para ver si respetan, o no, sus teorías proclamadas.

\subsection{Por qué proponemos diseñar a partir de principios de procedimiento}

Ya se ha expuesto que una de las tareas fundamentales a las que el profesorado se enfrenta es a la de planificar actividades, para lo cual se ha de tener en mente, a qué 
contenidos quiere acercar a su alumnado. Pero lo realmente difícil e importante, no es el qué, sino cómo va a acercarlo a dichos contenidos. El diseño didáctico es el eje central para el aprendizaje y, a este respecto, los objetivos educativos no sirven de guía para el profesorado, ya que el cómo garantizar que la actividad que le propongo a mis estudiantes sea potente, no tiene nada que ver con el qué debe mostrar el alumnado después de haberla realizado. La calidad del diseño de una actividad viene dada por cómo se organiza esta y bajo qué criterios, es decir, de qué cualidades educativas posee intrínsecamente dicha actividad. Blanco expone (1994):

Los profesores y profesoras, cuando seleccionamos los contenidos, los recursos o decidimos cómo queremos enseñar y qué criterios regirán la evaluación, no lo hacemos de modo secuencializado y parcializado. Tenemos presupuestos, concepciones, ideas acerca de lo que es valioso y posible, de cuál es el sentido y función de la escuela y de nuestro papel en ella, de lo que desearíamos y de lo que podemos hacer en unas circunstancias determinadas, que afectan a todos estos aspectos y que difícilmente pueden separarse; de tal modo que los contenidos que hayamos de enseñar están estableciendo parámetros para decidir cómo enseñarlos, con qué materiales, etcétera; pero también pensamos que hay ciertos materiales o recursos que son potencialmente valiosos y que llevan a matizar qué contenidos seleccionaremos; la manera en que entendamos la función y la naturaleza del conocimiento que enseñamos va a influir en las estrategias metodológicas para hacerlo accesible a los estudiantes, en el tipo de relaciones que hay que establecer en el aula, en procedimientos de evaluación, y así en muchos otros matices y aspectos (p. 214).

Cabe preguntarse si los objetivos ayudan a valorar la calidad de los aprendizajes. Parece evidente que sí, desde una lógica técnica de la educación en la que se determina la calidad del proceso educativo a partir de la consecución de unos objetivos establecidos, en término de conducta. Sin embargo, esta lógica evaluativa basada en la comprobación de objetivos ha recibido muchas críticas (Gimeno Sacristán, 2010; Álvarez Méndez, 2001; Alcaraz Salarirche, 2014).

En primer lugar, porque deja de lado el proceso que lleva a la consecución o no de dichos objetivos y, en segundo lugar, porque no está tan claro que la expresión de una conducta tenga que ver con el aprendizaje. Así lo afirma Stenhouse (1987, p.114) haciendo referencia a la relación conductas-aprendizajes:

El análisis del contenido del currículum en los objetivos de la conducta no está de acuerdo con la naturaleza y la estructura del conocimiento, con la epistemología. No cabe reducir el conocimiento a conductas. Especialmente no puede expresarse en términos de rendimientos especificados de antemano, porque la función del conocimiento es, en contraste con la simple acumulación de hechos, no determinar la conducta sino liberarla.

De igual forma se pronuncia Álvarez Méndez (2001, p. 37) al plantear la manera en la que se valora la consecución de objetivos:

Los tests de rendimiento o los exámenes conocidos como tradicionales carecen de interés y de valor formativo porque no nos dicen nada que nos ayude a entender estos procesos. Las formas más habituales de examinar, y también de corregir, tampoco. Los malos resultados 
son, si acaso, indicios de que algo no funciona, pero no nos dicen nada sobre las causas que los provocan, que pueden ser muchas, y no todas debidas a negligencias o torpezas de quien aprende.

En contraposición, la evaluación debe estar fundamentada "en la recogida de información útil y relevante, que sirva para emprender espacios de mejora en los procesos de enseñanza-aprendizaje" (Alcaraz Salarirche, Fernández Navas y Sola, 2012, p. 27). Es decir, que tiene sentido educativo si indaga en la calidad de los procesos. Puesto que la enseñanza no causa el aprendizaje, es importante conocer y comprender pormenorizadamente los procesos, ya que es esto lo que puede permitir al profesorado una valoración más ajustada de aquellos elementos de la enseñanza que deben ser cambiados y mejorados, para conseguir unas condiciones que permitan potencialmente aprendizajes de más calidad. Es aquí donde vamos a proponer que los principios de procedimiento -en contraposición al modelo de objetivos educativos- tienen más que decir con respecto a la calidad de las actividades que diseñamos. Porque, aunque aparentemente, el modelo por objetivos está desfasado y es una estrategia de programación didáctica antigua, y ahora programamos por competencias; no estamos seguros de que tal y como este modelo se está traduciendo a la práctica, sea tan diferente de las programaciones por objetivos. Así lo sugiere Gimeno Sacristán (2008) al analizar el nuevo concepto de competencias:

Causa cierta perplejidad la facilidad con la que se ponen en circulación lenguajes y metáforas que nos arrastran a denominar de manera aparentemente nueva a aquello que hasta ese momento reconocíamos de otra forma. La misma sensación nos produce el entusiasmo con el que, al parecer, nos sumamos a un nuevo universo de formas de hablar acerca de preocupaciones que aparentan ser novedosas porque con anterioridad se las llamaba de otra manera (p. 9).

Es por ello que, consideramos importante recuperar la relevancia de los principios de procedimiento como estrategia para aportar calidad a los procesos que se dan en nuestras aulas a través de actividades prácticas; ya que varios son los trabajos que exponen las ventajas de diseñar actividades a través de principios de procedimiento en el quehacer docente: Raths (1971), Stenhouse (1987), Bruner (1972), Zabala (1993), Blanco (1994) o Salinas (2001).

Antes de continuar con este planteamiento, queremos destacar que el uso de los principios de procedimiento no constituye -únicamente- una manera distinta de realizar una programación didáctica, sino que es más bien un modo diferente de entender la enseñanza. Mientras que en el modelo de objetivos se entiende como una cuestión técnica que consiste en pre-especificar unos buenos resultados de aprendizaje, para luego comprobarlos; la lógica de los principios de procedimiento se basa en entender que en el diseño de actividades y la forma en la que el profesorado las llevan a cabo, están implícitos, también, unos contenidos a los que éste quiere acercar a su alumnado, pero la forma de hacerlo pone el acento en la calidad de las actividades que diseña y pone en práctica en su aula y no, en la valoración de productos o conductas. 
El asunto es, pues, tratar de pensar la enseñanza de un modo diferente al que plantea el modelo de objetivos y no solamente proponer otro modelo de programación o de evaluación (Salinas, 2001).

Los principios de procedimiento son modos de proceder, de actuar, que cada docente se propone a sí mismo. Es decir, hablamos de estrategias de acción que definen una finalidad educativa, establecen criterios de actuación y tienden el puente entre el currículum como proyecto y su posibilidad de llevarlo a la práctica (Blanco, 1994). Estos criterios o características que deben cumplir las actividades diseñadas por el profesorado, plasman sus intenciones educativas con claridad y funcionan como orientaciones para pensar, modificar o desarrollar las actividades que piense realizar con su alumnado.

Es necesario destacar que el giro fundamental que se produce en la concepción de la enseñanza con el uso de los principios de procedimiento, comienza con que estos criterios o principios hacen referencia a ciertos requisitos que deben cumplir las actividades y, por lo tanto, en última instancia el profesorado. Nada que ver con definir con precisión metas, conductas, etc., que debe exhibir nuestro alumnado.

Mientras que en el modelo de objetivos la responsabilidad del error cae, generalmente, sobre el alumnado; en el modelo basado en principios de procedimiento, cae sobre las decisiones tomadas por el profesorado.

Por otro lado, frente al modelo basado en productos, pensar en la docencia a través de principios de procedimiento implica que estamos frente a un modelo centrado en los procesos. Un modelo que fija la atención del profesorado en lo que vale la pena hacer en sus aulas. Justo lo contrario que el modelo de objetivos, que aleja la reflexión docente hacia la comprobación de conductas, tal y como explicábamos al principio de este artículo.

La principal virtud, a nuestro modo de ver, del trabajo con principios de procedimiento es que establece un marco valorativo claro en el que puede discutirse la calidad de las actividades, permitiendo al propio alumnado de magisterio, analizar la coherencia existente entre lo que en teoría van proclamando y lo que en la práctica van diseñando. Es decir, el trabajo a través de principios de procedimiento les exige que sea la teoría la que respalde las decisiones didácticas que van tomando y les permite hacerlo de forma autónoma, sin la necesidad constante de tener un referente externo que les explicite si el diseño de su actividad es el adecuado o no.

Decimos que establece un marco claro de valoración de la calidad de las actividades en tanto en cuanto, por ejemplo, tenemos un principio de procedimiento que dice que una actividad "es más gratificante que otra si permite al alumnado efectuar elecciones informadas para realizarla y reflexionar sobre las consecuencias de sus acciones" (Raths 1971, citado en Stenhouse, 1984, pp. 131-132). Las actividades que diseñemos y que deben cumplir este principio serán más valiosas que las que hagamos con este otro principio de procedimiento: "Una actividad de enseñanza es más rica si le 
proporciona a mi alumnado una manera clara de reproducir lo que aparece en el libro de texto" (Raths 1971, citado en Stenhouse, 1984, pp. 131-132). Es decir, la elección de uno de esos dos principios de procedimiento como guía autoimpuesta para el diseño de actividades tiene que ver con el conocimiento acumulado que tenemos sobre psicología, didáctica, metodología, etc., y también con una cierta cosmovisión acerca de la función social de la escuela, el papel del docente en la enseñanza y el aprendizaje, la naturaleza del conocimiento, el rol de la teoría, el papel de la formación universitaria, entre otros.

Esta es, a nuestro juicio, una de las principales virtudes del uso de principios de procedimiento, la continua conexión entre qué merece la pena hacer en el aula y el conocimiento y la teoría que sustentan nuestro campo de estudio como eje principal de esas decisiones.

\subsection{Por qué proponemos diseñar escenarios de aprendizajes reales}

Pensar en actividades. Esto es lo que pedimos al alumnado universitario cuando lo ponemos en situaciones en las que han de diseñar y desarrollar una actividad para un grupo de estudiantes de una escuela. Y lo hacemos porque tal y como planteábamos en los inicios de este artículo, las dificultades que tienen los/as estudiantes en formación a la hora de integrar los conceptos teóricos en su diseño de actividades, son muchas. Lo cual genera que se encuentren con grandes problemas cuando les llega el momento de diseñar, desarrollar y evaluar actividades educativas.

La situación anteriormente descrita -estudiantes de los Grados de Educación Infantil y Primaria con muchas dificultades para diseñar y desarrollar actividades acordes a la teoría que estudian- debería ser un elemento importante de análisis para valorar cómo se está desarrollando la docencia en las aulas de las Facultades de Educación. Más aún, cuando entre las competencias que el alumnado de los Grados en Educación Infantil y Primaria deben adquirir, según la ORDEN ECI/3854/2007 y la ORDEN $\mathrm{ECl} / 3857 / 2007$ por las que se regulan los requisitos para la verificación de los títulos universitarios oficiales que habiliten para el ejercicio de la profesión de Maestro en Educación Infantil y Maestro en Educación Primaria, se encuentran las siguientes (tabla 1):

Grado en Educación Infantil
Grado en Educación

Primaria 


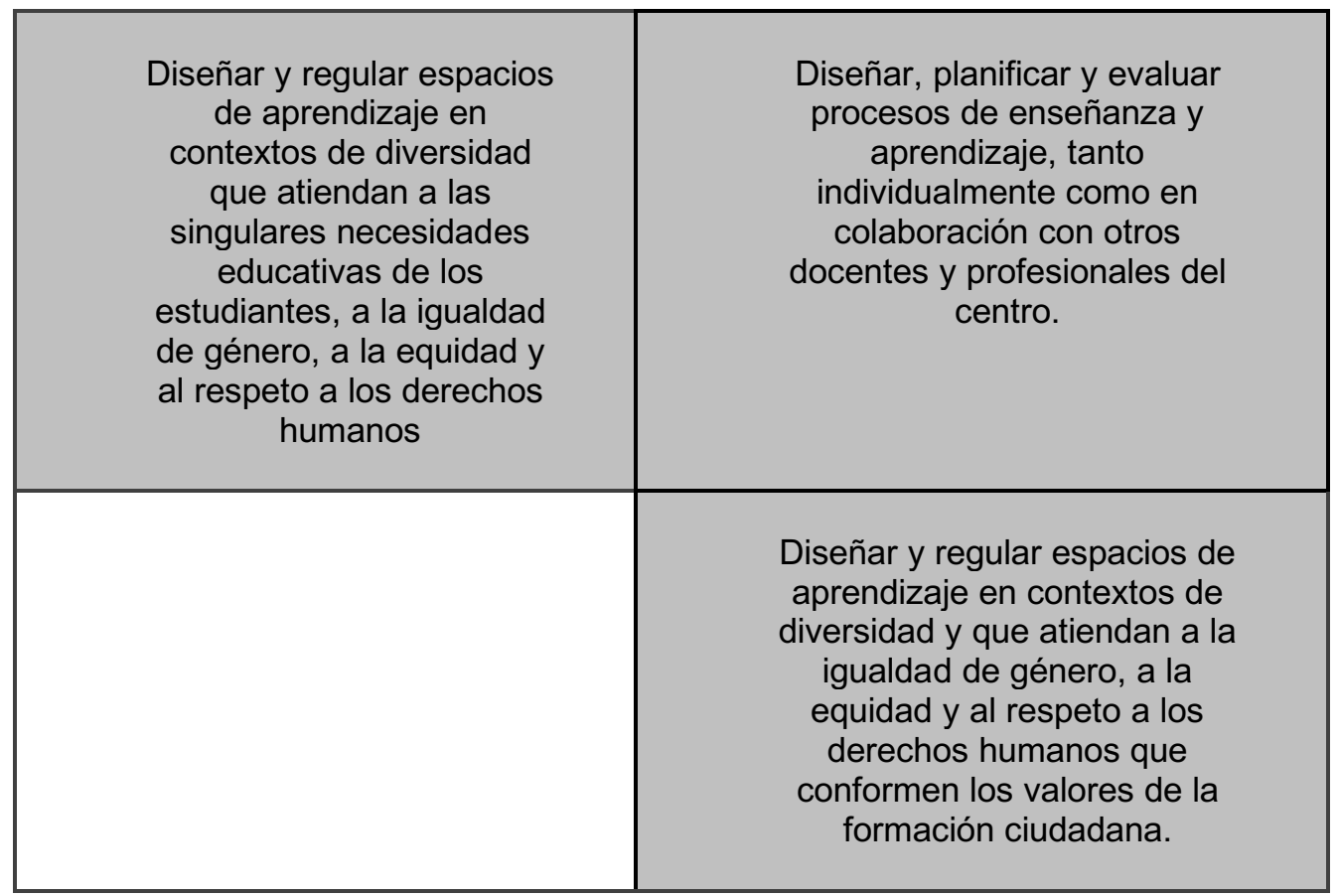

Tabla 1. Competencias que el alumnado de los Grados en Educación Infantil y Primaria deben adquirir. Fuente: BOE (2007) ORDEN ECI/3854/2007 y ORDEN ECI/3857/2007

Como se puede apreciar, el diseño y la planificación de espacios de enseñanzaaprendizaje, son competencias que el alumnado egresado de los Grados de Educación debería tener al finalizar su formación. Es por ello que nos parece urgente que el profesorado universitario trate de poner al alumnado en posibles situaciones prácticas del trabajo docente, pidiéndoles que usen la teoría para realizar esa tarea con más calidad. De modo que la teoría que les proporcionamos se convierta en una fuente de conocimiento útil, una herramienta, para resolver problemas en la práctica. $Y$ creemos que los principios de procedimiento, que venimos defendiendo, cobran un sentido valioso que les puede ayudar a integrar teoría y práctica.

La potencia formativa de este proceso de elaboración de principios de procedimiento radica en que el alumnado debe reconstruir su experiencia sobre las actividades. Y para ello, lo situamos en un punto clave para hacerlo con calidad: reflexionar en torno a qué implicaciones prácticas tienen los conceptos teóricos. Es en este punto de reflexión donde se produce una integración entre conceptos teóricos y prácticos, como diría Pérez Gómez (1998), una reconstrucción de la cultura experiencial a través de la cultura académica.

El alumnado en este punto tiene necesariamente que analizar las consecuencias prácticas que conllevan diferentes conceptos teóricos situándose obligatoriamente en un contexto de aplicación tal y como describe Bernstein (1995), y alejándolo del contexto de reproducción en el que normalmente lo situamos, con escasa o ninguna posibilidad de transformar las prácticas. 


\author{
Del mismo modo lo expresan Pérez Gómez, Soto, Sola, y Serván (2009) cuando \\ afirman:
}

Cuando como estudiantes o docentes nos proponemos desarrollar competencias tenemos que ampliar nuestra mirada y buscar no sólo el conocimiento de hechos o teorías (saber declarativo), sino también la operatividad y utilidad del mismo (saber hacer, saber expresar y saber comunicar), pero sobre todo el sentido que tienen dichos saberes para el desarrollo del propio proyecto personal y profesional (p. 19).

Esta manera de trabajar ayuda en dos aspectos fundamentales. Por un lado, está la reconstrucción de los conceptos en implicaciones prácticas sobre la que ya hemos hablado. Pero por otro -y no menos importante- es que desde su formación inicial les estamos ofreciendo un procedimiento que sirve para solventar problemas reales en la práctica y al que les acostumbramos a usar en nuestras aulas. De manera que cuando lleguen a su quehacer profesional, tengan experiencias en otro sentido sobre el diseño de actividades. Así como una "cartera" de principios de procedimiento con los que empezar a trabajar en la práctica. Principios de procedimiento basados y acordes al conocimiento de nuestro campo de estudio y que, creemos, ayuda a garantizar la calidad de las actividades que en un futuro diseñará el alumnado.

En definitiva, en los Grados de Educación inspirados en los principios del Espacio Europeo de Educación Superior, donde como plantea Esteban (2014, p. 5): "El estudiante ha adquirido un papel protagonista, siendo hacedor de su propio aprendizaje y constructor de su desarrollo académico, acompañado por un docente que tiene que redescubrir el conocimiento para acercarlo al estudiante de una forma nueva"; la apuesta por trabajar en situaciones prácticas con y a partir de principios de procedimientos, cobra más sentido que nunca. Necesitamos transformar nuestras aulas en espacios ricos donde el futuro profesorado descubra nuevas posibilidades a la hora de planificar actividades; lo cual pasa inevitablemente por ponerlo ante situaciones reales en las que tenga que tomar decisiones educativas a partir de esas otras posibilidades, por ejemplo, los principios de procedimiento.

\title{
3. Nuestra experiencia
}

Bajo la premisa de poner a nuestro alumnado de $2^{\circ}$ curso de la asignatura de Organización Educativa de la escuela y el aula de Infantil, ante una situación-problema que resolver; propusimos un reto: "Diseñar durante todo el semestre, por grupos, una actividad para llevar a cabo con un grupo de niños y niñas de Infantil que vendría a visitarnos a final de curso y con los cuales deberían trabajar toda la mañana realizando dicha actividad". Para ello, debían utilizar los contenidos de las asignaturas.

La única premisa era la de realizar una actividad con valor educativo basada en la metodología de trabajo por ambientes. Para lo cual les proponíamos que debían acudir a criterios psicológicos, pedagógicos, filosóficos y sociológicos trabajados y que debían 
respaldar teóricamente cada una de las decisiones tomadas sobre el diseño de su actividad. Es aquí donde entraban en juego los principios de procedimiento.

La primera fase del trabajo consistía en que cada grupo de estudiantes escogiera sus teorías y autores favoritos, con el propósito de fundamentar sus decisiones. Es decir, debían pensar en conceptos teóricos que sus actividades debían respetar. Una vez seleccionados estos conceptos, les decíamos que pensaran en las implicaciones que de ellos se derivaban para las actividades. Aspectos concretos y claros que les permitieran elaborar esos criterios que decíamos, y que constituyen los principios de procedimiento. Por ejemplo, si piensan que el concepto de atención a la diversidad que han trabajado en una de las asignaturas del Grado es algo valioso para su aula, les exponíamos que pensaran en posibles implicaciones de este concepto, como podría ser: "Todo el alumnado debe poder participar en igualdad de condiciones en las actividades que diseñe". Esta implicación práctica es lo que se convierte en uno de sus principios de procedimiento. Igual proceso debían realizar con todos los conceptos que consideraran valiosos hasta finalizar su lista de principios de procedimiento.

Es este proceso el que consideramos provechoso de cara a la formación inicial de docentes y así mismo, como ya argumentábamos anteriormente, la parte en el que el alumnado tiene más dificultades. Por ejemplo, la mayoría quiere incluir el aprendizaje significativo como concepto importante en sus actividades. No obstante, cuando les pedimos que planteen las implicaciones que este tiene para sus actividades, es cuando se bloquean. Y ahí es donde el profesorado universitario tiene un rol fundamental que consiste en ayudarles a reflexionar para que puedan ver las implicaciones prácticas de los conceptos teóricos. Hacerles ver que tener en cuenta el aprendizaje significativo implica, por ejemplo, criterios como: "Dejar en mis actividades un espacio al inicio para que el alumnado pueda hablar sobre lo que sabe y lo que no sabe del tema que estemos tratando; tener en cuenta que el tema que tratamos con nuestro alumnado sea asequible y comprensible para ellos; plantearles temas que les resulten interesantes para que la actividad favorezca interés, etc." Véase a continuación un ejemplo de teorías seleccionadas y principios de procedimiento que se derivan de ellas:

\begin{tabular}{|c|c|}
\hline \multicolumn{1}{|c|}{ Teorías } & \multicolumn{1}{|c|}{ Características de nuestras actividades } \\
\hline Conflicto cognitivo (Piaget) & $\begin{array}{l}\text {-Todas las actividades que diseñemos deben hacer dudar } \\
\text { al alumnado. }\end{array}$ \\
\hline Aprendizaje significativo (Ausubel) & $\begin{array}{l}\text {-Todas las actividades que diseñemos deben comenzar } \\
\text { con un espacio para activar los conocimientos previos de los } \\
\text { niños y las niñas. }\end{array}$ \\
\hline
\end{tabular}




\begin{tabular}{|c|c|}
\hline Zona de desarrollo próximo (Vigotsky) & $\begin{array}{l}\text {-Cuando formemos grupos con los niños y niñas debemos } \\
\text { crear grupos heterogéneos. } \\
\text {-En las actividades que diseñemos debemos plantearles } \\
\text { tareas cooperativas en las que deban ayudarse unos a otros. }\end{array}$ \\
\hline Aprender haciendo (Dewey) & $\begin{array}{l}\text {-Las actividades que planteemos deben ser prácticas y } \\
\text { permitir al alumnado experimentar. } \\
\text {-Debemos dar al alumno un papel activo en su aprendizaje. }\end{array}$ \\
\hline Etapas del desarrollo (Piaget) & $\begin{array}{l}\text {-Las actividades no deben requerir niveles de abstracción, } \\
\text { al contrario, deben ser manipulativas y favorecer la } \\
\text { exploración a través de los sentidos. }\end{array}$ \\
\hline Teoría del descubrimiento de Brunner & $\begin{array}{l}\text {-Actividades donde puedan actuar de forma autónoma y } \\
\text { descubrir por sí mismos la realidad que les rodea. }\end{array}$ \\
\hline Montessori & $\begin{array}{l}\text {-Usar materiales de la vida cotidiana } \\
\text {-Distribuirlos todos al alcance de los niños y las niñas. }\end{array}$ \\
\hline 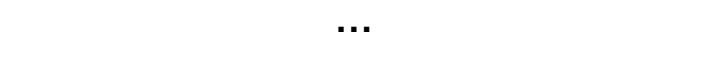 & \\
\hline
\end{tabular}

Tabla 2. Ejemplo de tabla con teorías y principios de procedimiento

Como se puede apreciar, no estamos más que plasmando las características especificadas por las teorías y sus autores y autoras, en implicaciones prácticas para las actividades. Este proceso de diseño al que sometemos al alumnado en formación, queda recogido en la figura 1. 
Concepto teórico

Deben decidir qué conceptos teóricos quieren trasladar a sus actividades

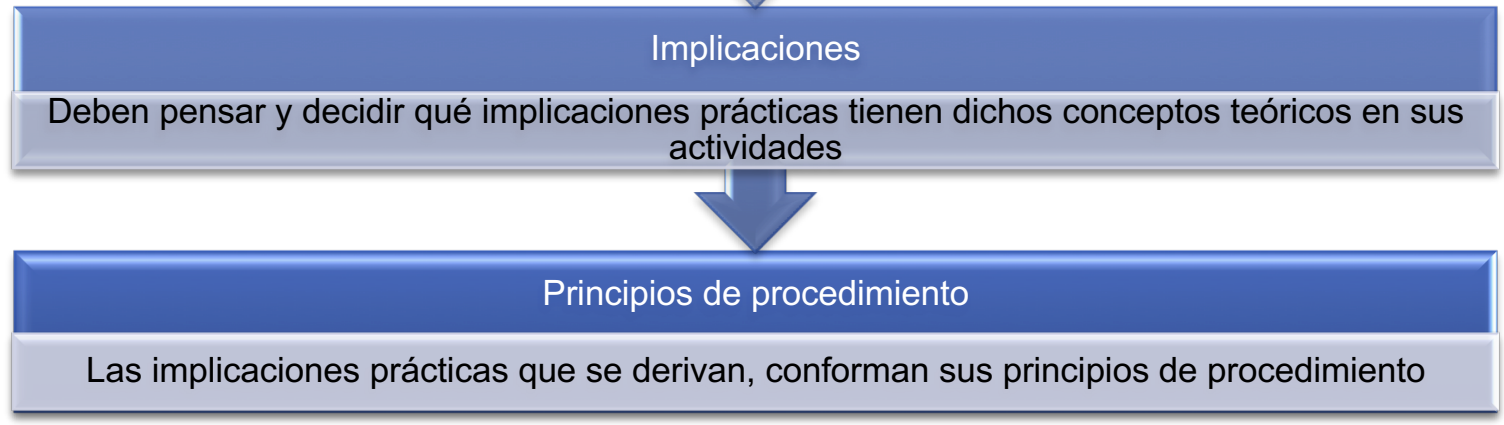

Figura 1. Proceso de diseño de los principios de procedimientos. Fuente: elaboración propia

Una vez que todos los grupos tuviesen sus principios de procedimiento realizados, llegaba la hora de preparar una actividad educativa real que respetara cada uno de dichos principios. Era el momento de ponerse manos a la obra, decidir qué y cómo trabajar con el grupo de niños y niñas que nos visitarían.

De modo que, durante la mitad del semestre cada grupo de estudiantes diseñó una propuesta didáctica basada en la metodología de trabajo por ambientes, creando un total de diez micro-espacios: Laberinto, mesa de luz, arenero, flotabilidad, cabaña de indios, música y color, cocina, recorrido de botellas, artístico y pizarra magnética. Poco a poco, las teorías y principios de procedimientos utilizados iban dando lugar a actividades concretas fundamentadas coherentemente y respaldadas normativamente. Véanse como ejemplo las tablas 3 y 4. 


\begin{tabular}{|c|c|c|c|}
\hline Teorías & $\begin{array}{c}\text { Características de } \\
\text { nuestras actividades }\end{array}$ & $\begin{array}{l}\text { Actividades a } \\
\text { realizar }\end{array}$ & $\begin{array}{c}\text { Orientación } \\
\text { metodológica de la } \\
\text { normativa }\end{array}$ \\
\hline $\begin{array}{l}\text { Aprender } \\
\text { haciendo } \\
\text { (Dewey) }\end{array}$ & $\begin{array}{l}\text { Las actividades que } \\
\text { planteemos deben ser } \\
\text { prácticas y permitir al } \\
\text { alumnado } \\
\text { experimentar. } \\
\text { Debemos dar al } \\
\text { alumno/a un papel } \\
\text { activo en su } \\
\text { aprendizaje. }\end{array}$ & $\begin{array}{l}\text { Vamos a diseñar } \\
\text { un micro-espacio } \\
\text { con materiales no } \\
\text { estructurados y } \\
\text { elementos } \\
\text { naturales como } \\
\text { cuerdas, madera, } \\
\text { telas, plumas, } \\
\text { etc., para que el } \\
\text { alumnado pueda } \\
\text { construir una } \\
\text { cabaña de forma } \\
\text { autónoma. }\end{array}$ & $\begin{array}{c}\text { Enfoque } \\
\text { globalizador y } \\
\text { aprendizaje } \\
\text { significativo. } \\
\text { La actividad infantil, } \\
\text { la observación y la } \\
\text { experimentación. } \\
\text { Los espacios y los } \\
\text { materiales: Soporte } \\
\text { para la acción, } \\
\text { interacción y } \\
\text { comunicación. }\end{array}$ \\
\hline
\end{tabular}

Tabla 3. Ejemplo de ampliación de la tabla de teorías y principios de procedimiento

\section{Nombre de la actividad: La cabaña de los indios}

Descripción: Se pondrán a disposición de los niños y las niñas los materiales necesarios para construir una cabaña. El espacio en el que llevaremos a cabo la actividad será en los exteriores de la facultad. Todo ello, se trabajará en un entorno natural donde se fomentará la manipulación, la experimentación y la acción mental a través del juego. Esta actividad está planteada para que al niño o a la niña le suponga un reto estimulante su realización y al mismo tiempo favorecer la motivación intrínseca.

Justificación de la temática: Hemos decidido llevar a cabo esta actividad porque la consideramos idónea para trabajar en ambientes puesto que los niños y las niñas trabajan con autonomía en el transcurso de la actividad sin seguir las directrices del adulto; el adulto solo se muestra como una figura de acompañamiento o mediación en caso de que el alumnado necesite ayuda 
para la realización de la actividad. Además, es una actividad motivadora ya que potencia la creatividad y la imaginación de los niños y de las niñas, puesto que, en estas edades también es importante crear actividades que conecten con el mundo de fantasía de los niños y de las niñas.

Finalidades: Con esta actividad pretendemos potenciar la motricidad fina, concretamente, la coordinación óculo-manual; el aprendizaje cooperativo, la cohesión grupal y el intercambio de ideas a través de la interrelación entre los niños y las niñas. Otra de las finalidades es garantizar la autonomía durante el transcurso de la actividad respetando la individualidad de cada alumno/a y los ritmos de aprendizaje.

\section{Áreas del currículum:}

1. Conocimiento de sí mismo y autonomía personal

2. Conocimiento del entorno

3. Lenguajes: comunicación y representación

Materiales: Tablones de madera, plumas, cuerdas, sábanas, tiras de velcro con adhesivo a doble cara, pinturas para la cara 2 paquetes, cojines, 2 paquetes de bolsas de basura, cartulina roja, amarilla y cartón.

Tabla 4. Ejemplo de ficha de actividad de un grupo

Este proceso resultó muy valioso desde el punto de vista de la vinculación entre práctica y teoría, ya que nuestro papel de tutorización se centró en plantearles preguntas acerca de las decisiones que iban tomando. Por ejemplo: ¿Por qué habéis decidido agrupar a los niños por niveles? ¿Por qué vais a realizar una asamblea inicial? ¿Por qué no utilizáis materiales naturales? ¿Por qué vais a dejar que jueguen? Tratábamos de que fuesen capaces de argumentar cada actividad y decisión que iban tomando. Para ello les hacíamos recurrir a sus principios de procedimiento. De este modo sus explicaciones eran cada vez más justificadas y argumentadas pedagógicamente:

Se trataba de diseñar ambientes de aprendizaje que contribuyeran de forma significativa al crecimiento integral de los niños. Nuestro micro-espacio les permitía tomar decisiones donde poder probar distintas estrategias, resolver problemas por sí mismos, pensar y descubrir, aprender del fracaso y del error y utilizar esos resultados. Por ejemplo, en el momento de montar la tienda de campaña, antes de atarle la cuerda a los palos, casi siempre la soltaban y cuando veían los palos en el suelo, se daban cuenta realmente que hacía falta algo más para sujetarlos (Ejemplo de justificación de una alumna).

Por último, como en cualquier práctica, no todo lo que hacemos sale como pretendemos. Es aquí donde resultó valiosa una tercera fase, la reflexión sobre cómo transcurrieron las actividades. A este respecto, cada grupo debía planificar una evaluación in-situ que permitiera analizar si en sus actuaciones estaban siendo respetuosos con sus principios. Para ello se plantearon una lista de focos o cuestiones donde poner la atención. Por 
ejemplo: ¿Se ajustan las actividades al tiempo estipulado? ¿Da lugar la actividad a un trabajo cooperativo? ¿Ha ayudado la presentación del material? ¿Debemos volver a limitar el espacio? ¿Hemos organizado bien los tiempos? ¿Hemos sido muy directivas? ¿Hemos sido demasiado permisivas? Veamos a continuación un ejemplo de tabla donde debían ir registrando información a través de la observación de la actividad (imagen 1).

\begin{tabular}{|l|l|l|l|}
\hline \multicolumn{3}{|c|}{ AUTOEVALUACIÓN INTEGRANTES DEL AMBIENTE } \\
\hline $\begin{array}{l}\text { ¿Cómo actuamos ante un problema? } \\
\text { ¿Debemos hacerlo más de una vez? }\end{array}$ & & & \\
\hline $\begin{array}{l}\text { ¿Cómo intervenimos? ¿Tenemos que } \\
\text { improvisar? Si es así, ¿nos sale bien?! }\end{array}$ & & & \\
\hline $\begin{array}{l}\text { ¿Hemos organizado bien el material y } \\
\text { el espacio? }\end{array}$ & & & \\
\hline ¿Cuánto tiempo observamos? & & & \\
\hline $\begin{array}{l}\text { ¿Qué método seguimos para } \\
\text { observar? }\end{array}$ & & & \\
\hline ¿Hicimos preguntas a los niños? & & & \\
\hline ¿Qué es lo que funcionó o no? & & & \\
\hline $\begin{array}{l}\text { ¿Qué es lo que hemos querido recoger } \\
\text { (primera visita)? }\end{array}$ & & & \\
\hline
\end{tabular}

Imagen 1. Ejemplo de autoevaluación de un grupo

A través de este proceso de observación y reflexión compartida una vez transcurrida la actividad, los estudiantes fueron descubriendo algunas disonancias entre lo que pretendían hacer y lo que hicieron de verdad, es decir, detectaron incoherencias entre sus principios de procedimiento y sus modos de proceder en la práctica. Así, por ejemplo, algunos grupos tenían entre sus principios "servir de guía", tras la observación de los compañeros y la puesta en común posterior, pudieron analizar qué significa realmente ser un "guía" y cómo, aunque ellos pretendían teóricamente cumplir con dicha premisa, a la hora de la verdad se descubrían siendo excesivamente directivos con los niños. Fue este proceso el que dio lugar al ajuste de algunos principios de procedimiento y a la mejora organizativa de ciertas cuestiones detectadas, con el fin de volver a llevar acabo la actividad mejorada en una segunda visita de los Colegios.

\section{En definitiva...}


Entendemos que de esta forma se ajustan las disonancias entre la praxis y la teoría, entre el conocimiento declarado y la acción, a través de un proceso de indagación sobre la propia práctica.

Como hemos visto a lo largo de este trabajo, el modelo de programación didáctica tal y como está planteado a través de objetivos, no ayuda al profesorado a realizar actividades de más calidad con su alumnado. Y, por tanto, el tiempo que invertimos en su formación inicial a trabajar estas programaciones se convierte en una vía muerta hacia el diseño de actividades de calidad.

Creemos que el trabajo a través de principios de procedimiento, tal y como hemos expuesto, sitúa al futuro profesorado en una manera de diseñar actividades dirigida hacia la calidad de las mismas y hacia la coherencia con el saber acumulado en educación. La programación de actividades cuyas cualidades intrínsecas sean de verdadera calidad educativa es la única garantía de que el alumnado de los Grados en Educación consiga hacer aprendizajes de calidad.

Consideramos que situar a los/as estudiantes ante el problema de diseñar estos principios, es una tarea que merece la pena en tanto en cuanto les obliga a reflexionar en torno a las implicaciones prácticas de los conceptos teóricos que ven en su formación. Y esta reflexión les permite reconstruir su experiencia sobre actividades a través de un conocimiento probado y elaborado sobre educación, cumpliendo con el papel activo y autónomo que debe tener el alumnado y que es reclamado desde la filosofía de las titulaciones de los nuevos Grados.

Además, creemos que es de gran valor que se acostumbren a trabajar en este sentido en las aulas universitarias para ofrecerles un modelo alternativo de pensar en actividades y para que cuando se incorporen a su práctica tengan experiencias en otro sentido sobre el diseño de las mismas. Así como un abanico amplio de principios de procedimiento sólidos con los que empezar a trabajar y, por supuesto, a repensar y re-elaborar a lo largo de su carrera docente.

\section{Bibliografía}

\section{PUBLICACIONES PERIÓDICAS}

Esteban, R., M., (2014). El nuevo estudiante en el Espacio Europeo de Educación Superior. Tendencias Pedagógicas, 23, 5-6.

Gertrudix, S. (1999). La enseñanza programada. Aula libre, 69, 1, 22-26. 
Pérez Gómez, A. I., Soto, E. y Serván Ma José (2015). Lesson Studies: Re-pensar y re-crear el coocimiento práctico en cooperación. Revista Interuniversitaria de Formación del Profesorado, 84, 81-101.

Pérez Granados, L. (2018). El grupo de trabajo como estrategia de formación permanente del profesorado. Aula de Encuentro, 20, 1, 4-25. DOI: https://dx.doi.org/10.17561/ae.v20i1.1

Raths , J. (1971). Teaching without specific objectives. Educational Leadership, 28, 714-20.

Zabala, A. (1992). Elaboración de los PCC. ¿Empezar por lo que se hace o partir de las grandes decisiones? Aula de innovación educativa, 3, 60-66.

\section{LIBROS Y CAPÍTULOS DE LIBRO}

Álvarez Méndez, J. (2001). Evaluar para conocer, examinar para excluir. Madrid: Morata

Argyris, C. (1993). Knowledge for action: A guide to overcoming barriers to organizational change. San Francisco: Jossey-Bass.

Bernstein, B. (1995). A response. In A. R. Sadovnik (comp.) Knowledge and pedagogy: the sociology of Basil Bernstein (pp. 385-424). New Jersey: Norwood, Ablex Publishing.

Blanco, N. (1994). Las intenciones educativas. In J. Ángulo, \& N. Blanco (coords.) Teoría y desarrollo del currículum (pp. 205-231). Málaga: Aljibe.

Bruner, J. (1972). Hacia una teoría de la instrucción. Barcelona: Ed. Uteha.

Contreras, J. (2001). La autonomía del profesorado. Madrid: Morata

Gimeno Sacristán, J. (Comp.) (2008). Educar por competencias, ¿qué hay de nuevo? Madrid: Morata.

Gimeno Sacristán, J. (2010). ¿Qué significa el currículum? In J. Gimeno Sacristán (Ed.), Saberes e incertidumbres del currículum (pp. 19-44). Madrid: Morata.

Imbernón, F. (2010). La formación del profesorado y el desarrollo del currículum. In J. Gimeno Sacristán (Ed.), Saberes e incertidumbres sobre el currículum (pp. 588-603). Madrid: Morata.

Pérez Gómez A. (1998). La cultura escolar en la sociedad neoliberal. Madrid: Morata.

Pérez Gómez, A., Sola, M., Blanco, N., \& Barquín, J. (2004). Luces y sombras en la situación profesional de los docentes españoles. En Gimeno Sacristán, J. \& Carbonell, J. El sistema educativo. Una mirada crítica (pp. 121-145). Barcelona: Praxis. 
Pérez Gómez, A., Soto, E., Sola, M., \& Serván, M. (2009). Aprender cómo aprender Autonomía y responsabilidad: el aprendizaje de los estudiantes. Guía $\mathrm{n}^{\circ} 3$ Espacio Europeo de Educación Superior. Madrid: Akal.

Salinas, D. (2001). ¡Mañana examen! La evaluación: entre la teoría y la realidad. Barcelona: Graó.

Schön, D. A. (1987). La formación de profesionales reflexivos. Hacia un diseño de la enseñanza y el aprendizaje en las profesiones. Barcelona: Paidós

Sierra, E. Y Caparrós, E. (2017). Apuntes acerca de la formación de educadoras y educadores y el desarrollo de la interioridad. In Martín \& Castilla (coords.). Aproximaciones a las complejidades de la Paz (pp. 69-76). Granada: GEU.

Stenhouse, L. (1984). Investigación y desarrollo del currículum. Madrid: Morata.

Stenhouse, L. (1987). La investigación como base de la enseñanza. Madrid: Morata.

\section{MEDIOS ELECTRÓNICOS}

Alcaraz Salarirche, N., Fernández Navas, M., \& Sola, M. (2012). La voz del alumnado en los procesos de evaluación docente universitaria. Revista Iberoamericana de Evaluación Educativa, 5, 2, 26-39. (http://www.rinace.net/riee/numeros/vol5-num2/art2.pdf)

Alcaraz Salarirche, N. (2014). Un viejo trío de conceptos: aprendizaje, currículum y evaluación. $\begin{array}{lllrr}\text { Aula de } & \text { Encuentro, } & 16, & 2 . & 55-86\end{array}$ (http://revistaselectronicas.ujaen.es/index.php/ADE/article/view/1771)

BOE (1990). Ley Orgánica 1/1990, de 3 de octubre de 1990, de Ordenación General del Sistema Educativo (https://boe.es/diario boe/txt.php?id=BOE-A-1990-24172)

BOE (2007). ORDEN ECI/3854/2007 de Maestro en Educación Infantil (https://www.boe.es/diario boe/txt.php?id=BOE-A-2007-22446)

BOE (2007) ORDEN ECI/3857/2007 de Maestro en Educación Primaria (https://www.boe.es/diario boe/txt.php?id=BOE-A-2007-22449)

\section{REFERENCIA BIBLIOGRÁFICA}


Alcaraz Salarirche, N., Fernández Navas, M. y Pérez Granados, L. (2019) Principios de procedimiento y escenarios reales en la formación inicial de docentes. Aula de Encuentro, $\mathrm{n}^{\circ} 21$, volumen 1 , pp. 123-142.

Noelia Alcaraz Salarirche

Profesora del Dpto. de Didáctica y Organización Escolar de la Universidad de Málaga

Correo-e: noe@uma.es

Manuel Fernández Navas

Profesor del Dpto. de Didáctica y Organización Escolar de la Universidad de Málaga Correo-e: mfernandez1@uma.es

Laura Pérez Granados Profesora del Dpto. de Didáctica y Organización Escolar de la Universidad de Málaga Correo-e: Ipgranados@uma.es 